\title{
Line gracefulness of some cycle related graphs
}

\author{
S K Vaidya ${ }^{1}$, N J Kothari ${ }^{2}$ \\ ${ }^{1}$ (Department of Mathematics, Saurashtra University Rajkot - 360005, Gujarat, INDIA.) \\ ${ }^{2}$ (Atmiya Institute of Technology and Science, Rajkot-360005, Gujarat, India.)
}

\begin{abstract}
We investigate line graceful labeling for the spliting graph, total graph, shadow graph and mirror graph of cycle. Moreover we prove that the graphs obtained by duplication of each edge of cycle by a vertex and duplication of each vertex of cycle by an edge admit line graceful labeling.
\end{abstract}

Keywords: Graceful labeing, edge graceful labeling, line graceful labeling, total graph, spliting graph, shadow graph.

AMS Subject Classification (2010): 05C78, 05C38, $05 C 76$.

\section{Introduction}

Labeling of discrete structures is a one of the potential areas of research due to its diversified applications. According to Prasanna et al. [1] graph labeling plays vital role in expanding the utility of the channel assignment process in communication network. The optimal linear arrangement concern to network problems in electrical engineering and placement problems in production engineering can be formalized as a graph labeling problems as stated by Yegnanaryanan and Vaidhyanathan [2]. A dynamic survey on different graph labeling schemes with an extensive bibliography can be found in Gallian [3]. The graphs considered here are finite, connected and undirected without loops and multiple edges. For standard terminology and notation we refer to West [4].

Definition 1.1: A graph labeling is an assignment of numbers to the vertices or edges or both subject to certain condition(s).

Definition 1.2: A function $f$ is called graceful labeling of graph if $f: V(G) \rightarrow\{0,1,2,3, \ldots, q\}$ is injective and the induced function $f^{*}: E(G) \rightarrow\{1,2, \ldots, q\}$ defined as $f^{*}(e=u v)=|f(x)-f(y)|$ is bijective. A graph which admits graceful labeling is called a graceful graph.

Most of the graph labeling techniques trace their origin with the graceful labeling which was introduced independently by Rosa [5] and Golomb [6]. A variant of graceful labeling termed as edge graceful labeling has been introduced by Lo [7].

Definition 1.3: A graph $G=(V(G), E(G))$ is said to be edge graceful if there exists a bijection $f: E(G) \rightarrow\{1,2,3, \ldots, q\}$ such that the induced mapping $f^{*}: V(G) \rightarrow\{0,1, \ldots, p-1\}$ defined by $f^{*}(v)=\sum_{v v_{i} \in E(G)} f\left(v v_{i}\right)(\bmod p)$ is bijection.

Lo [7] have also derived a necessary condition for a graph with $p$ vertices and $q$ edges to be edgegraceful is $q(q+1) \equiv \frac{p(p+1)}{2}(\bmod p)$ and also investigate edge graceful labeling of various graph families. All trees of odd order are edge graceful as conjunctured by Lee [8]. Many graphs are proved to be edge graceful by Lee et al. $[9,10,11]$. The edge gracefulness in the context of cartesian product of odd cycles is studied by Wilson and Risking [12]. A variant of edge graceful labeling termed as line graceful labeling has been introduced by Gnanajothi [13] in her Ph.D. thesis.

Definition 1.4: A mapping $f: E(G) \rightarrow\{0,1,2, \ldots, p\}$ is called line graceful of graph with $p$ vertices, if induced function $f^{*}: V(G) \rightarrow\{0,1,2, \ldots, p-1\}$ defined by $f^{*}(v)=\sum_{v v_{i} \in E(G)} f\left(v v_{i}\right)(\bmod p)$ is bijective.

Vaidya and Kothari [14,15] have discussed line gracefulness in the context of switching of vertex operation, graphs arising from different graph operations on path while this paper is aimed to discuss line gracefulness in 
the context of graph operations on cycle and some standard graphs. We will give brief summary of definitions which are essential for the present investigations.

Definition 1.5: For every vertex $v \in V(G)$, the open neighbourhood set $N(v)$ is the set of vertices which are adjacent to $v$ in $G$.

Definition 1.6: For a graph $G$ the splitting graph $S^{\prime}(G)$ of graph $G$ is obtained by adding a new vertex $v^{\prime}$ corresponding to each vertex $v$ of $G$ such that $N(v)=N\left(v^{\prime}\right)$.

Definition 1.7: The total graph $T(G)$ of a graph $G$ is the graph whose vertex set is $V(G) \cup E(G)$ and two vertices are adjacent whenever they are either adjacent or incident in $G$.

Definition 1.8: The shadow graph $D_{2}(G)$ of a connected graph $G$ is obtained by taking two copies of $G$, say $G^{\prime}$ and $G^{\prime \prime}$ and joining each vertex $u^{\prime}$ of $G^{\prime}$ to the neighbours of corresponding vertex $u^{\prime}$ of $G^{\prime \prime}$.

Definition 1.9: Let $G$ be a bipartite graph with a partite sets $V_{1}$ and $V_{2}$ and $G^{\prime}$ be the copy of $G$ with corresponding partite sets $V_{1}^{\prime}$ and $V_{2}^{\prime}$. The mirror graph of $G$ is obtained from $G$ and $G^{\prime}$ by joining each vertex of $V_{1}$ to its corresponding vertex in $V_{1}^{\prime}$ by an edge.

Definition 1.10:[16] Duplication of an edge $e=u v$ by a new vertex $w$ in a graph $G$ produces a new graph $G^{\prime}$ such that $N(w)=\{u, v\}$.

Definition 1.11:[16] Duplication of a vertex $v_{i}$ by a new edge $e=v_{i}^{\prime} v_{i}^{\prime \prime}$ in graph $G$ produces a new graph $G^{\prime}$ such that $N\left(v_{i}^{\prime}\right) \cap N\left(v_{i}^{\prime \prime}\right)=\left\{v_{i}\right\}$.

\section{Main results}

Proposition 2.1[13]: If the graph is line graceful then its order is not congruent to $2(\bmod 4)$.

This necessary condition serves as a sufficient condition for the graph which does not admit line graceful labeling.

Theorem 2.2: $S^{\prime}\left(C_{n}\right)$ is line graceful only for $n \equiv 0,2(\bmod 4)$.

Proof: Let $v_{1}, v_{2}, \ldots, v_{n}$ be the vertices of cycle $C_{n}$ and $u_{1}, u_{2}, \ldots, u_{n}$ be the vertices corresponding to $v_{1}, v_{2}, \ldots, v_{n}$ which are added to obtain $S^{\prime}\left(C_{n}\right)$. We note that $\left|V\left(S^{\prime}\left(C_{n}\right)\right)\right|=2 n$ and $\left|E\left(S^{\prime}\left(C_{n}\right)\right)\right|=3 n$. Define edge labeling $f: E\left(S^{\prime}\left(C_{n}\right)\right) \rightarrow\{0,1, \ldots, 2 n\}$ as follows.

Case 1: $n \equiv 0,2(\bmod 4)$

for $1 \leq i \leq n-1$

$f\left(v_{i} v_{i+1}\right)= \begin{cases}0 & \text { for odd } i \\ n & \text { for even } i\end{cases}$

$f\left(u_{i} u_{i+1}\right)=i \quad$ for $1 \leq i \leq n-1$

$f\left(u_{i} v_{i-1}\right)=0 \quad$ for $2 \leq i \leq n$

$f\left(u_{1} v_{n}\right) \quad=0$

$f\left(v_{n} v_{1}\right)=f\left(u_{n} v_{1}\right)=n$

Above defined edge labeling function satisfies the condition for line graceful labeling.

Case 2: $n \equiv 1,3(\bmod 4)$

In this case $\left|V\left(S^{\prime}\left(C_{n}\right)\right)\right|=2 n \equiv 2(\bmod 4)$.

Then according to Proposition $2.1 S^{\prime}\left(C_{n}\right)$ is not line graceful.

In view of above defined edge labeling function will induce the bijective vertex labeling function $f^{*}: V\left(S^{\prime}\left(C_{n}\right)\right) \rightarrow\{0,1, \ldots, 2 n-1\}$ such that $f^{*}(v)=\sum_{e \in E\left(S^{\prime}\left(C_{n}\right)\right)} f(e)(\bmod 2 n)$ for $n \equiv 0,2(\bmod 4)$. Hence $S^{\prime}\left(C_{n}\right)$ is line graceful graph only for $n \equiv 0,2(\bmod 4)$. 
Example 2.3: $S^{\prime}\left(C_{n}\right)$ and its line graceful labeling is shown in Fig.1

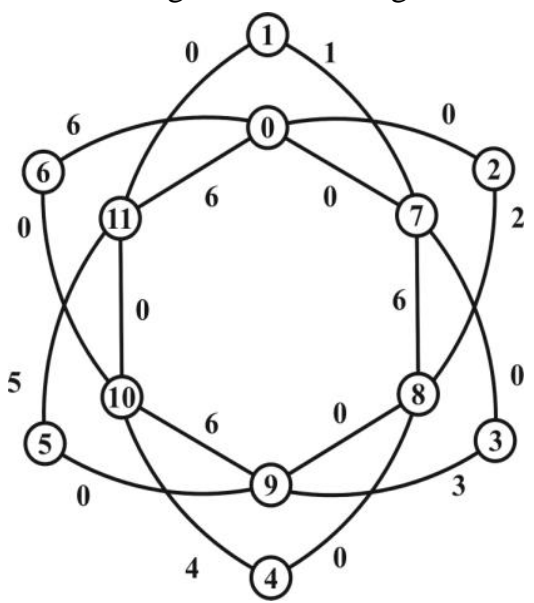

Fig. 1

Theorem 2.4: $T\left(C_{n}\right)$ is line graceful only for $n \equiv 0(\bmod 2)$.

Proof: Let $v_{1}, v_{2}, \ldots, v_{n}$ be the vertices and $e_{1}, e_{2}, \ldots, e_{n}$ be the edges of cycle $C_{n}$. Then $V\left(T\left(C_{n}\right)\right)=V\left(C_{n}\right) \cup E\left(C_{n}\right)$ and $E\left(T\left(C_{n}\right)\right)=E\left(C_{n}\right) \bigcup\left\{v_{i} e_{i} ; 1 \leq i \leq n, v_{i} e_{i-1} ; 2 \leq i \leq n, v_{1} e_{n}, e_{i} e_{i+1} ; 1 \leq i \leq n-1, e_{n} e_{1}\right\}$. We note that $\left|V\left(T\left(C_{n}\right)\right)\right|=2 n$ and $\left|\mathrm{E}\left(\mathrm{C}_{n}\right)\right|=4$ n. Define edge labeling $f: E\left(T\left(C_{n}\right)\right) \rightarrow\{0,1, \ldots, 2 n\}$ as follows.

Case 1: $n \equiv 0(\bmod 2)$

for $1 \leq i \leq n-1$

$f\left(v_{i} v_{i+1}\right)= \begin{cases}0 & \text { for odd } i \\ n & \text { for even } i\end{cases}$
$f\left(u_{i} u_{i+1}\right)=0 \quad$ for $1 \leq i \leq n-1$
$f\left(u_{n} u_{1}\right)=0$
$f\left(u_{i} v_{i+1}\right)=i \quad$ for $1 \leq i \leq n-1$
$f\left(u_{i} v_{i}\right)=0 \quad$ for $1 \leq i \leq n$
$f\left(v_{n} v_{1}\right)=f\left(u_{n} v_{1}\right) \quad=n$

Above defined edge labeling function satisfies the condition for line graceful labeling.

Case 2: $n \equiv 1(\bmod 2)$

In this case $\left|V\left(T\left(C_{n}\right)\right)\right|=2 n \equiv 2(\bmod 4)$.

Then according to Proposition $2.1 T\left(C_{n}\right)$ is not line graceful.

In view of above defined edge labeling function will induce the bijective vertex labeling function $f^{*}: V\left(T\left(C_{n}\right)\right) \rightarrow\{0,1, \ldots, 2 n-1\}$ such that $f^{*}(v)=\sum_{e \in E\left(T\left(C_{n}\right)\right)} f(e)(\bmod 2 n)$ for $n \equiv 0(\bmod 2)$. Hence $T\left(C_{n}\right)$ is line graceful graph only for $n \equiv 0(\bmod 2)$.

Example 2.5: $T\left(C_{n}\right)$ and its line graceful labeling is shown in Fig. 2

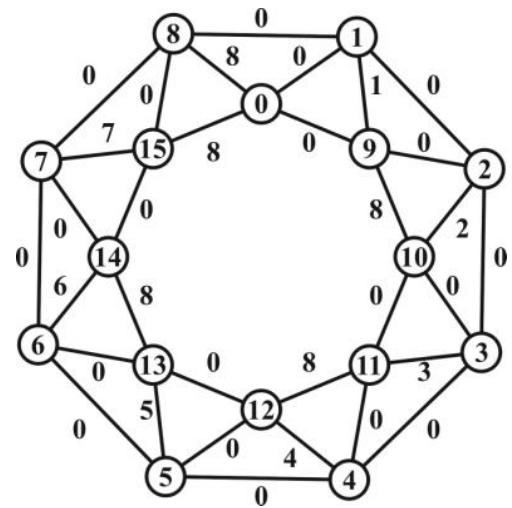

Fig. 2 
Theorem 2.6: $D_{2}\left(C_{n}\right)$ is line graceful only for $n \equiv 0(\bmod 2)$.

Proof: Consider two copies of cycle $C_{n}$. Let $v_{1}, v_{2}, \ldots, v_{n}$ be the vertices of first copy of cycle $C_{n}$ and $u_{1}, u_{2}, \ldots, u_{n}$ be the vertices of second copy of cycle $C_{n}$. Hence $V\left(D_{2}\left(C_{n}\right)\right)=\left\{v_{1}, v_{2}, \ldots, v_{n}, u_{1}, u_{2}, \ldots, u_{n}\right\}$ and $E\left(D_{2}\left(C_{n}\right)\right)=E\left(C_{n}\right) \cup\left\{u_{i} u_{i+1} ; 1 \leq i \leq n-1, u_{n} u_{1}, u_{i} v_{i+1} ; 1 \leq i \leq n-1, u_{n} v_{1}, u_{i} v_{i-1} ; 2 \leq i \leq n, u_{1} v_{n}\right\}$. We note that $\left|V\left(D_{2}\left(C_{n}\right)\right)\right|=2 n$ and $\left|E\left(D_{2}\left(C_{n}\right)\right)\right|=4 n$. Define edge labeling $f: E\left(D_{2}\left(C_{n}\right)\right) \rightarrow\{0,1, \ldots, 2 n\}$ as follows.

Case 1: $n \equiv 0(\bmod 2)$

for $1 \leq i \leq n-1$

$$
\begin{aligned}
& f\left(v_{i} v_{i+1}\right)= \begin{cases}1 & \text { for odd } \mathrm{i} \\
0 & \text { for even } \mathrm{i}\end{cases} \\
& f\left(u_{i} u_{i+1}\right)=0 \quad \text { for } 1 \leq i \leq n-1 \\
& f\left(u_{i} v_{i+1}\right)=2 i \text { for } 1 \leq i \leq n-1 \\
& f\left(u_{i} v_{i-1}\right)=0 \quad \text { for } 2 \leq i \leq n \\
& f\left(v_{n} v_{1}\right)=f\left(u_{n} u_{1}\right)=f\left(u_{n} v_{1}\right)=f\left(u_{1} v_{1}\right)=0
\end{aligned}
$$

Above defined edge labeling function satisfies the condition for line graceful labeling.

Case 2: $n \equiv 1(\bmod 2)$

In this case $\left|V\left(D_{2}\left(C_{n}\right)\right)\right|=2 n \equiv 2(\bmod 4)$.

Then according to Proposition $2.1 D_{2}\left(C_{n}\right)$ is not line graceful.

In view of above defined edge labeling function will induce the bijective vertex labeling function $f^{*}: V\left(D_{2}\left(C_{n}\right)\right) \rightarrow\{0,1, \ldots, 2 n-1\}$ such that $f^{*}(v)=\sum_{e \in E\left(D_{2}\left(C_{n}\right)\right)} f(e)(\bmod 2 n)$ for $n \equiv 0(\bmod 2)$. Hence $D_{2}\left(C_{n}\right)$ is line graceful graph only for $n \equiv 0(\bmod 2)$.

Example 2.7: $D_{2}\left(C_{n}\right)$ and its line graceful labeling is shown in Fig. 3 .

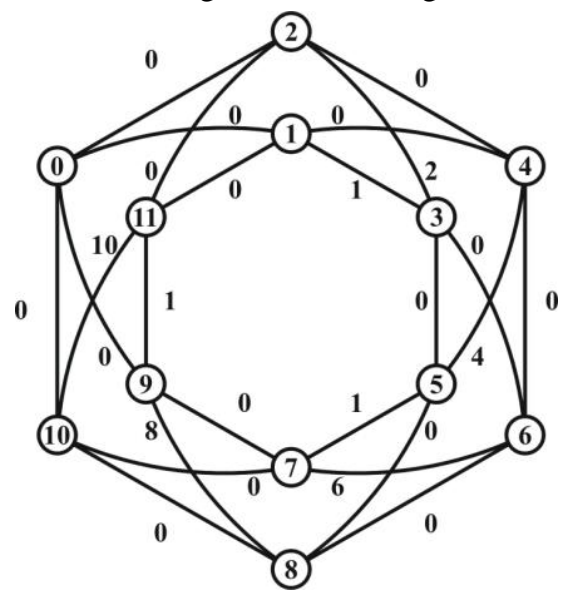

Fig. 3

Theorem 2.8: Mirror graph of even cycle $C_{n}$ is line graceful.

Proof: Consider two copies of cycle $C_{n}$. Let $v_{1}, v_{2}, \ldots, v_{n}$ be the vertices of first copy of cycle $C_{n}$ and $u_{1}, u_{2}, \ldots, u_{n}$ be the vertices of second copy of cycle $C_{n}$, were $n$ is even. Let $V_{1}=\left\{v_{1}, v_{3}, \ldots, v_{n-1}\right\}, V_{2}=\left\{v_{2}, v_{4}, \ldots, v_{n}\right\}$ be the partite sets of first copy of $C_{n}$ and $U_{1}=\left\{u_{1}, u_{3}, \ldots, u_{n-1}\right\}, U_{2}=\left\{u_{2}, u_{4}, \ldots, u_{n}\right\}$ be the partite sets of second copy of $C_{n}$. The mirror graph $G$ of cycle $C_{n}$ is obtained by joining each vertex of $V_{1}$ to its corresponding vertex in $U_{1}$. Hence $V(G)=\left\{v_{1}, v_{2}, \ldots, v_{n}, u_{1}, u_{2}, \ldots, u_{n}\right\}$ and $E(G)=E\left(C_{n}\right) \bigcup\left\{u_{i} u_{i+1} ; 1 \leq i \leq n-1, u_{n} u_{1}, u_{i} v_{i} ; 1 \leq i \leq n-1\right.$ for odd $\left.i\right\}$. We note that $|V(G)|=2 n$ and $|E(G)|=2 n+\frac{n}{2}$.

Define edge labeling $f: E(G) \rightarrow\{0,1, \ldots, 2 n\}$ as follows.

for $1 \leq i \leq n-1$ 


$$
\begin{aligned}
& f\left(v_{i} v_{i+1}\right)= \begin{cases}n+i & \text { for odd } \mathrm{i} \\
0 & \text { for even } \mathrm{i}\end{cases} \\
& f\left(v_{n} v_{1}\right)=f\left(u_{n} u_{1}\right) \quad=0 \\
& \text { for } 1 \leq i \leq n-1 \\
& f\left(u_{i} u_{i+1}\right)= \begin{cases}i & \text { for odd } \mathrm{i} \\
0 & \text { for even } \mathrm{i}\end{cases} \\
& f\left(u_{i} v_{i}\right) \quad=2 n-1 \quad \text { for odd } \mathrm{i}
\end{aligned}
$$

Above defined edge labeling function satisfies the condition for line graceful labeling.

In view of above defined edge labeling function will induce the bijective vertex labeling function $f^{*}: V(G) \rightarrow\{0,1, \ldots, 2 n-1\}$ such that $f^{*}(v)=\sum_{e \in E(G)} f(e)(\bmod 2 n)$ for $n \equiv 0(\bmod 2)$. Hence mirror graph of even cycle is line graceful.

Example 2.9: Mirror graph of cycle and its line graceful labeling is shown in Fig. 4

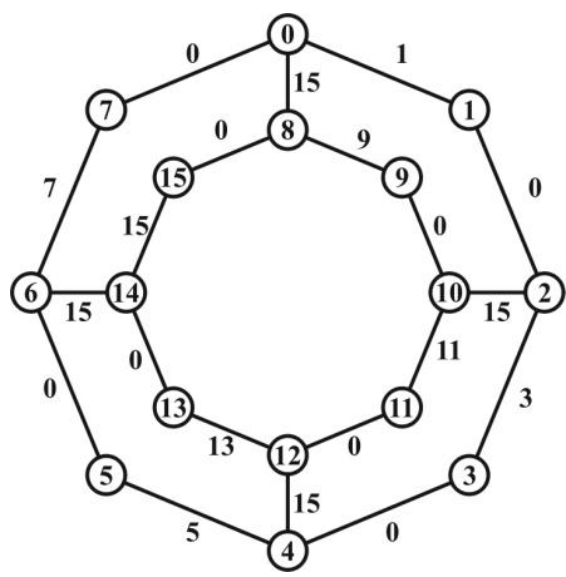

Fig. 4

Theorem 2.10: The graph obtained by duplication of each vertex of $C_{n}$ by an edge admits line graceful labeling except $n \equiv 2(\bmod 4)$.

Proof: Let $v_{1}, v_{2}, \ldots, v_{n}$ be the vertices of cycle $C_{n}$ and $G$ be the graph obtained by duplication of each vertex $v_{i}$ of cycle $C_{n}$ by an edge $u_{i} u_{i+1}(1 \leq i \leq n)$.

Then $V(G)=V\left(C_{n}\right) \bigcup\left\{u_{1}, u_{2}, \ldots, u_{2 n}\right\}$ and $E(G)=E\left(C_{n}\right) \bigcup\left\{u_{2 i-1} v_{i}, u_{2 i} v_{i}, u_{i} u_{i+1} ; 1 \leq i \leq 2 n-1\right.$ for odd $\left.i\right\}$. We note that $|V(G)|=3 n$ and $|E(G)|=4 n$.

Define edge labeling $f: E(G) \rightarrow\{0,1, \ldots, 2 n\}$ as follows.

Case 1: $n \equiv 0,1(\bmod 4)$

$$
\begin{aligned}
& f\left(v_{i} v_{i+1}\right) \quad=3\left\lfloor\frac{i}{2}\right\rfloor \quad \text { for } 1 \leq i \leq n-1 \\
& f\left(v_{n} v_{1}\right) \quad=0 \\
& f\left(u_{2 i-1} v_{i}\right)=1 \quad \text { for } 1 \leq i \leq n-1 \\
& f\left(u_{2 n-1} v_{n}\right)=\left\lfloor\frac{3 n-f\left(v_{n-1} v_{n}\right)}{2}\right\rfloor \\
& f\left(u_{2 i} v_{i}\right) \quad=2 \quad \text { for } 1 \leq i \leq n-1 \\
& f\left(u_{2 n} v_{n}\right) \quad=\left\lceil\frac{3 n-f\left(v_{n-1} v_{n}\right)}{2}\right\rceil \\
& f\left(u_{i} u_{i+1}\right) \quad=3(i-1) \quad \text { for } 1 \leq i \leq 2 n-3, i \equiv 1(\bmod 2) \\
& f\left(u_{2 n-1} u_{2 n}\right)=3 n-1-f\left(u_{2 n} v_{n}\right)
\end{aligned}
$$

Case 2: $n \equiv 3(\bmod 4)$

$$
f\left(v_{1} v_{2}\right)=0
$$

for $1 \leq i \leq n-1$ 


$$
\begin{aligned}
& f\left(v_{i} v_{i+1}\right)= \begin{cases}3 n-\frac{3 i}{2} & \text { for even } \mathrm{i} \\
f\left(v_{i-1} v_{i}\right) & \text { for odd } \mathrm{i}\end{cases} \\
& f\left(u_{2 i-1} v_{i}\right)=3 i-2 \quad \text { for } 1 \leq i \leq n-1 \\
& f\left(u_{2 n-1} v_{n}\right)=\left\lfloor\frac{3 n-f\left(v_{n-1} v_{n}\right)}{2}\right\rfloor \\
& f\left(u_{2 i} v_{i}\right) \quad=3 i-1 \quad \text { for } 1 \leq i \leq n-1 \\
& f\left(u_{2 n} v_{n}\right) \quad=\left\lceil\frac{3 n-f\left(v_{n-1} v_{n}\right)}{2}\right\rceil \\
& f\left(u_{2 i-1} u_{2 i}\right)=0 \quad \text { for } 1 \leq i \leq n-1, i \equiv 1(\bmod 2) \\
& f\left(u_{2 n-1} u_{2 n}\right)=3 n-2-f\left(u_{2 n-1} v_{n}\right)
\end{aligned}
$$

Above defined edge labeling function satisfies the condition for line graceful labeling.

Case 3: $n \equiv 2(\bmod 4)$

In this case $|V(G)|=3 n \equiv 2(\bmod 4)$.

Then according to Proposition $2.1 G$ is not line graceful.

In view of above defined edge labeling function will induce the bijective vertex labeling function $f^{*}: V(G) \rightarrow\{0,1, \ldots, 3 n-1\}$ such that $f^{*}(v)=\sum_{e \in E(G)} f(e)(\bmod 3 n)$ for $n \equiv 0,1,3(\bmod 4)$. Hence we proved that $G$ is line graceful graph except for $n \equiv 2(\bmod 4)$.

Example 2.11: Graph obtained by duplication of vertex of $C_{n}$ by an edge and its line graceful labeling is shown in Fig. 5.

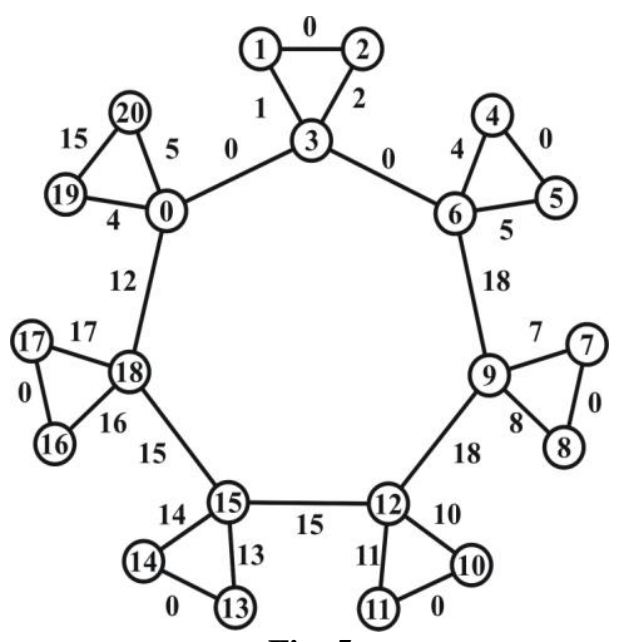

Fig. 5

Theorem 2.12: The graph obtained by duplication of an edge of $C_{n}$ by a vertex admits line graceful labeling only for $n \equiv 0(\bmod 2)$.

Proof: Let $v_{1}, v_{2}, \ldots, v_{n}$ be the vertices of cycle $C_{n}$ and $G$ be the graph obtained by duplication of an edge $v_{i} v_{i+1}$ by a vertex $u_{i}(1 \leq i \leq n)$. Then $V(G)=V\left(C_{n}\right) \bigcup\left\{u_{1}, u_{2}, \ldots u_{n}\right\}$ and $E(G)=E\left(C_{n}\right) \bigcup\left\{u_{i} v_{i} ; 1 \leq i \leq n, u_{i} v_{i+1} ; 1 \leq i \leq n-1\right.$, $\left.u_{n} v_{1}\right\}$. We note that $|V(G)|=2 n$ and $|E(G)|=3 n$. Define edge labeling $f: E(G) \rightarrow\{0,1, \ldots, 2 n-1\}$ as follows.

Case 1: $n \equiv 0(\bmod 2)$

for $1 \leq i \leq n-1$

$$
\begin{aligned}
& f\left(v_{i} v_{i+1}\right)= \begin{cases}0 & \text { for odd } \mathrm{i} \\
n & \text { for even } \mathrm{i}\end{cases} \\
& f\left(u_{i} v_{i+1}\right)=i \quad \text { for } 1 \leq i \leq n-1 \\
& f\left(u_{i} v_{i}\right)=0 \quad \text { for } 1 \leq i \leq n
\end{aligned}
$$

Above defined edge labeling function satisfies the condition for line graceful labeling. 
Case 2: $n \equiv 1(\bmod 2)$

In this case $|V(G)|=2 n \equiv 2(\bmod 4)$.

Then according to Proposition $2.1 G$ is not line graceful.

Thus above defined edge labeling function will induce a bijective vertex labeling function $f^{*}: V(G) \rightarrow\{0,1, \ldots, 2 n-1\}$ such that $f^{*}(v)=\sum_{e \in E(G)} f(e)(\bmod 2 n)$ for $n \equiv 0(\bmod 2)$. Hence, $G$ is line graceful graph only for $n \equiv 0(\bmod 2)$.

Example 2.13: The graph obtained by duplication of an edge of $C_{n}$ by a vertex and its line graceful labeling is shown in Fig. 6.

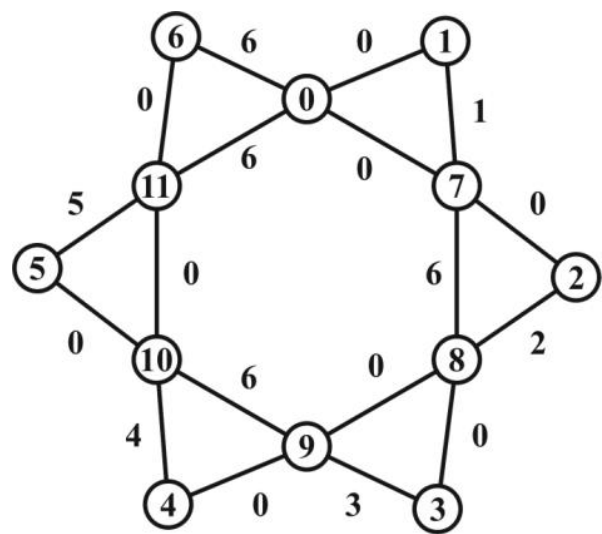

Fig. 6

\section{Conclusion}

Edge gracefulness and line gracefulness of a graph are two independent concepts. A graph may possess one or both of these or neither as mentioned below.

1. $C_{2 n+1}$ is edge graceful as well as line graceful.

2. $P_{n}$ is neither edge graceful nor line graceful for $n \equiv 2(\bmod 4)$.

3. $C_{4 n}$ is not edge graceful but line graceful.

4. Triangular snake $T_{n}$ is edge graceful only for $n=3$ while it is line graceful for all $n$.

\section{Acknowledgement}

The authors are highly thankful to the anonymous referees for their kind suggestions and comments.

\section{References}

[1] N. Lakshmi Prasanna K. Sravanthi and Nagalla Sudhakar, Applications of Graph Labeling in Communication Networks, Oriental Journal of Computer science \& Technology, 7(1), 2014, 139-145.

[2] V. Yegnanaryanan and P. Vaidhyanathan, Some Interesting Applications of Graph Labelings, J. Math. Comput. Sci., 2(5), 2012, $1522-1531$.

[3] J. A. Gallian, A dynamic survey of graph labeling, The Electronic Journal of Combinatorics, 16(2013), \#DS6.

[4] D. B. West, Introduction to Graph Theory (2/e, Prentice-Hall of India, 2003, New Delhi).

[5] A. Rosa, On certain valuations of the vertices of a graph, in Theory of Graphs, International Symposium Rome, (July 1966), Gordon and Breach), N.Y. and Dunod Paris, 1967, 349-355.

[6] S.W. Golomb, How to number a graph, in R C Read, (Ed.), Graph Theory and Computing, (New York: Academic Press, 1972) 2337.

[7] S. Lo, On edge-graceful labelings of graphs, Congr. Numer., 50, 1985, 231-241.

[8] S. M. Lee, A conjecture on edge-graceful trees, Scientia Series A: Math. Sciences, 3, 1989, 45-47.

[9] Q. Kuang, S. M. Lee, J. Mitchem and A-G. Wang, On edge-graceful unicyclic graphs, Congr. Numer., 61, $1988,65-74$.

[10] S. M. Lee and E. Seah, Edge-gracefulness labelings of regular complete K-partite graphs, Congr. Numer., 75, 1990, 41-50.

[11] K. Schaffer and S. M. Lee, Edge-graceful and edge-magic labelings of cartesian products of graphs, Congr. Numer., 141, 1999, $119-134$.

[12] S. Wilson and A. Riskin, Edge-graceful labelings of odd cycles and their products, Bulletin of the ICA, $24,1998,57-64$.

[13] R. B. Gnanajothi, Topics in Graph Theory, Ph.D. Thesis, Madurai Kamaraj University, Tamil Nadu(India), 1991.

[14] S. K. Vaidya and N. J. Kothari, Some new families of line graceful graphs, International Journal of Mathematics and Scientific Computing, 1(2), 2011, 26-28.

[15] S. K. Vaidya and N. J. Kothari, Line Gracefulness of Some Path Related Graphs, International Journal of Mathematics and Scientific Computing, 4(1), 2014, 15-19.

[16] S.K.Vaidya and Lekha Bijukumar, Some New Families of Mean Graphs, Journal of Mathematics Research, 2(3), 2010, $169-176$. 